\title{
Strategy for Switchover Between the Ground Return and the Metallic Return Operation Modes of MTDC Systems
}

\author{
Manxi Huang \\ North China Electric Power \\ University \\ Beijing, China \\ 18577105086@163.com
}

\author{
Hengyi Pan \\ North China Electric Power \\ University \\ Beijing, China \\ 1791173400@qq.com
}

\author{
Gen Li \\ Cardiff University \\ Cardiff, England \\ LiG9@cardiff.ac.uk
}

\author{
Jianzhong Xu \\ North China Electric Power \\ University \\ Beijing, China \\ xujianzhong@ncepu.edu.cn
}

\author{
Xiufang Jia \\ North China Electric Power \\ University \\ Beijing, China \\ xiufangjia@163.com
}

\begin{abstract}
For a multi-terminal HVDC (MTDC) system in the bipolar configuration with earth electrodes, the switchover between the ground return operation mode and the metallic return operation mode is underresearched. An optimal strategy for switchover needs to be selected to minimize the interrupting capacities of the metallic return transfer breaker (MRTB) and the ground return transfer switch (GRTS) so as to reduce capital costs. To select an optimal strategy for switchover between the two operation modes of MTDC systems, the paper not only develops location selection schemes and switching sequences of MRTB and GRTS but also proposes a concise and feasible method to address the maximums of the interrupting current functions of MRTB and GRTS under every scheme and sequence using the random number calculation method. In the paper, based on a three-terminal HVDC system, numerical examples using MATLAB have proved the effectiveness of the proposed method of selecting an optimal strategy for switchover between the two operation modes.
\end{abstract}

Keywords- Multi-terminal HVDC (MTDC), Metallic return transfer breaker (MRTB), Ground return transfer switch (GRTS), Random number calculation.

\section{INTRODUCTION}

HIGH-VOLTAGE direct-current (HVDC) transmission system in bipolar configuration is suitable for transferring bulk power with a high reliability due to its monopolar operation capability [1]-[2]. Earth electrode is commonly used in bipolar HVDC systems to carry ground return currents and allows for asymmetrical monopole operation [3]. Generally, the earth electrode and its associated metallic line are called the earth electrode system (hereinafter referred to as the earth electrode). In case of a malfunction of converters or during the maintenance of one pole, the other pole can still operate with the help of the ground return or the metallic return [4]. However, a long period of operation with the ground return may result in a corrosive effect to the soil near the earth electrode of the converter [5]-[7]. Hence, it is advisable to temporarily use the other pole as the conductor instead of using the ground as the current return path [8], which is illustrated in Fig. 1.

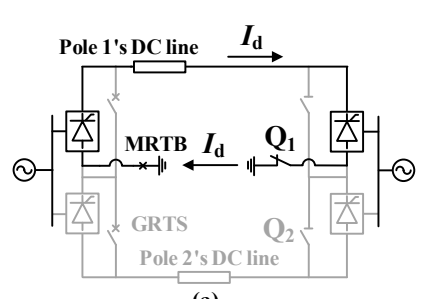

(a)

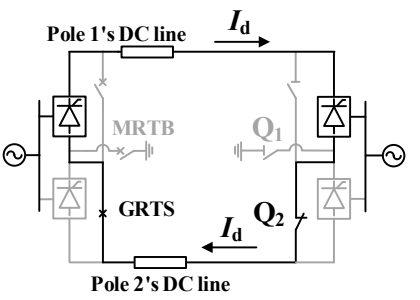

(b)
Fig. 1. Monopolar operation modes. (a) Using ground return, (b) Using the other pole.

To switch over between the above two operation modes, the ground and the other pole (as a temporary metallic return) will be firstly put into parallel [9]. Due to the low resistance of the ground, the majority of the DC current will flow in the ground return. To switch from the ground return to the metallic return, the metallic return transfer breaker (MRTB) will be opened. To switch from the metallic return to the ground return, the ground return transfer switch (GRTS) will be opened [10]. These two switchgears have been used in the line commuted converter (LCC) based HVDC links.

The multi-terminal HVDC (MTDC) system that can balance and increase the utilization of various power resources is becoming popular [11]-[13]. The Kun-Liu-Long three-terminal ultra HVDC network has one LCC station and two modular multilevel converter (MMC) stations. The system is a bipolar configuration with earth electrodes and therefore, when converters in one pole fail or in maintenance, the healthy pole can operate with the help of the ground return or the other pole (metallic return) [14]-[15]. To improve operational reliability and flexibility, a bipolar MTDC system 
with earth electrodes requires a feasible strategy to switch over between the two operation modes. However, the switchover has some new features which should be considered in designing the switchover strategy:

1) MRTB and GRTS need to be installed in more than one converter station.

2) The switchover should be carried out in a proper sequence of the operations of MRTB and GRTS because there are communication delays among stations and it takes time for the system to reach the steady-state after the switching of the switchgears.

3) The set-up locations of the MRTB and GRTS in the converter stations in the MTDC will affect their interrupting currents because the geographic locations and capacity of each station are different. The interrupting currents of MRTB and GRTS also vary under different switching sequences of them because the switching operation in one station may change the currents in the return circuits of other stations.

When selecting the MRTB and GRTS in the design stage, a smaller interrupting current means a smaller interrupting capacity and fewer capital costs. An optimal strategy for switchover between operation modes of MTDC can result in minimum interrupting currents of MRTB and GRTS. Therefore, the optimal strategy for switchover between operation modes should consider the deployment and switching sequence of MRTB and GRTS. However, there is no such optimal strategy in the open literature.

In [16]-[17], design suggestions for MRTB and GRTS are proposed. The testing principle and method of MRTB are proposed in [18]. However, references [16]-[18] only study the performance of the switchgears without considering their optimal system-level operation and coordination. A fast switchover method has been studied in [19], which can only reduce the time for the switchover scheme but cannot reduce the interrupting currents of the switchgears. The current distribution between GRTS and MRTB is obtained by simulations in [20]. However, the study can only be effective under a specific switching sequence. Reference [21] analyses two switching sequences based on a three-terminal HVDC network, but involves neither all switching sequences nor different deployment schemes of MRTB and GRTS.

In order to select an optimal strategy for switchover between the ground return and metallic return modes of MTDC systems, the paper: (i) develops clear definitions of all possible deployment schemes and switching sequences of MRTB and GRTS; (ii) proposes a method to address the maximum interrupting currents of MRTB and GRTS using random number calculations. Using this method, maximum interrupting currents of MRTB and GRTS of every deployment scheme and switching sequence can be obtained. Minimum interrupting currents can be achieved thanks to the proposed optimal strategy. Based on a three-terminal HVDC network, numerical examples using MATLAB and simulations in PSCAD/EMTDC show that the proposed methods are effective and feasible.

\section{Models For Switchover BetweEn the Ground RETURN AND METALLIC RETURN MODES OF MTDC SYSTEMS}

This section presents models for analyzing the switchover between the ground return and metallic return modes for MTDC systems. Based on the models, deployment schemes and switching sequences of MRTB and GRTS are illustrated.

A. Models for Analyzing the Switchover Between Two Modes

Taking the hybrid LCC/MMC MTDC system as an example, radial and ring MTDC systems in bipolar configuration are illustrated in Fig. 2. It is worth mentioning that the used models are not restricted by the type of converters. MRTB is installed in the earth electrode circuit of the converter, and GRTS is installed between the earth electrode and the pole (in parallel with the converter). MRTB is used to transfer the DC current from an earth return path to a metallic return path without interrupting the DC power flow, and GRTS does the opposite. GRTS must be used in combination with MRTB [9]. It needs two stations to establish a return circuit. However, as long as one station has an MRTB/GRTS, the return circuit can be cut off. As a result, it is not necessary to set up MRTB and GRTS at every station.

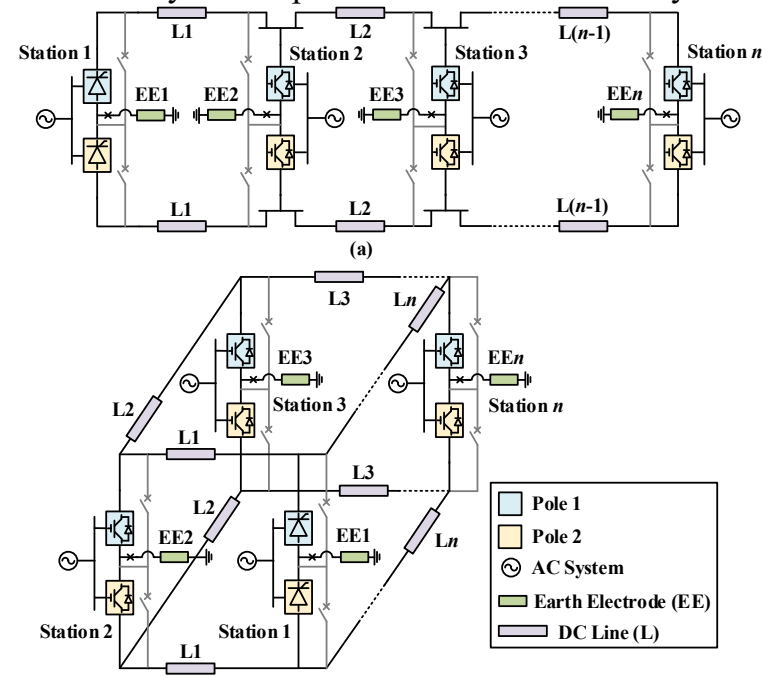

Fig. 2. MTDC systems in bipolar configuration. (a) Radial, (b) Ring.

Based on Fig. 2, models for analyzing the switchover between the two operation modes of MTDC systems are illustrated in Fig. 3. In this case, Pole 2 is out of service due to malfunction or maintenance (Pole 2 is omitted), and Pole 1 will operate in the monopolar ground return mode. Pole 1 is desirable to switch to the metallic return mode, and then transfer back to the ground return mode once Pole 2 recovers. 


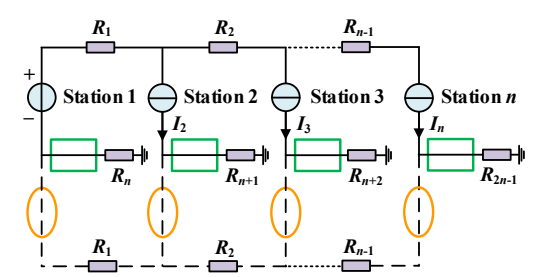

(a)

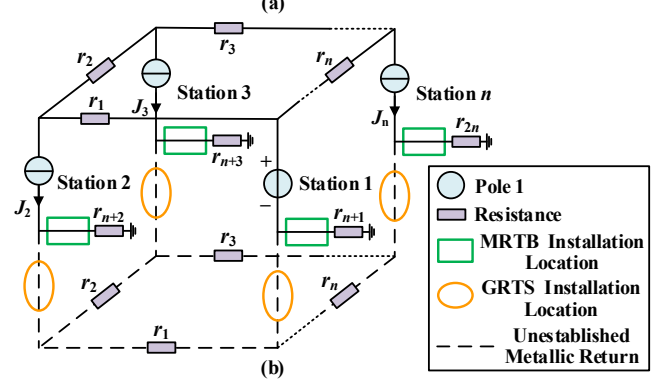

Fig. 3. Models for analyzing the switchover of MTDC. (a) Radial, (b) Ring.

In Fig. 3, unestablished metallic returns (DC lines of Pole 2) are shown as dashed lines, and the possible set-up location of MRTB is indicated by a box and GRTS by a circle. Under steady-state operation, any $(n-1)$ stations of an $n$-terminal DC system can be equivalent to current sources. In Fig. 3(a), DC outputs of Station 2, Station 3, .., Station $n$ are defined as $I_{2}$, $I_{3}, \ldots, I_{n}$, resistances of DC lines are defined as $R_{1}, R_{2}, \ldots, R_{n-}$ 1, and resistances of earth electrodes are defined as $R_{n}, R_{n+1}$, $\ldots, R_{2 n-1}$. In Fig. 3(b), DC outputs of Station 2, Station 3, .., Station $n$ are defined as $J_{2}, J_{3}, \ldots, J_{n}$, resistances of DC lines are defined as $r_{1}, r_{2}, \ldots, r_{n}$, and resistances of earth electrodes are defined as $r_{n+1}, r_{n+2}, \ldots, r_{2 n}$.

\section{B. Deployment Schemes and Switching Sequences of $M R T B$ and GRTS}

Based on Fig. 3, to switch over the operation mode of Pole 1, MRTB and GRTS need to be set up at $(n-1)$ stations, and the remaining station only needs disconnectors. Table I summarizes $n$ deployment schemes of MRTB and GRTS for both radial and ring MTDC systems. Theoretically, the deployment scheme of MRTB could be different from GRTS. However, to facilitate management and operation, MRTB and GRTS are usually set up at the same station. When the ground return and metallic return circuits are put into parallel, due to the low resistance of the ground, the ground return circuit carries most of the DC current. Consequently, the demand for interrupting capacity of MRTB is higher, and MRTB is given higher priority. Once the set-up location of MRTB is determined, the set-up location of GRTS will be determined.

TABLE I

\begin{tabular}{cc}
\multicolumn{2}{c}{ DEPLOYMENT SCHEMES OF MRTB AND GRTS FOR MTDC SYSTEMS } \\
\hline \hline $\begin{array}{c}\text { Deployment } \\
\text { Schemes }\end{array}$ & Stations with MRTB and GRTS \\
\hline $\begin{array}{cc}\text { Scheme } 1 \\
\text { Scheme } 2\end{array}$ & Station 1, Station $2, \ldots$, Station $(n-1)$ \\
$\ldots$ & Station 1, Station $2, \ldots$, Station $(n-2)$, Station $n$ \\
Scheme $n$ & $\ldots$ \\
\hline \hline
\end{tabular}

As illustrated in Fig. 4, taking Scheme 1 as an example, MRTB and GRTS are set up at Station 1, Station 2, ..., Station $(n-1)$, and Station $n$ is equipped with disconnectors (Q) only. In Fig. 4(a), MRTB and GRTS are numbered from left to right, while MRTB and GRTS are numbered clockwise in Fig. 4(b).

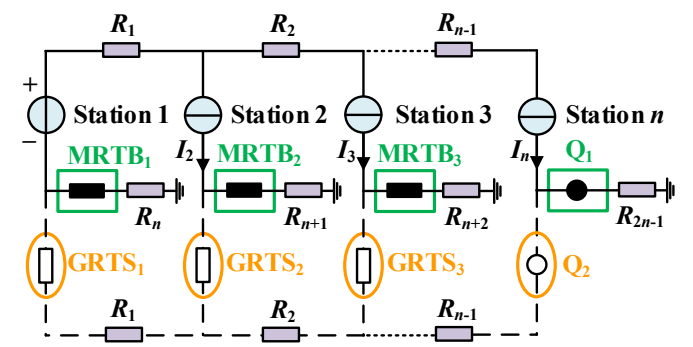

(a)

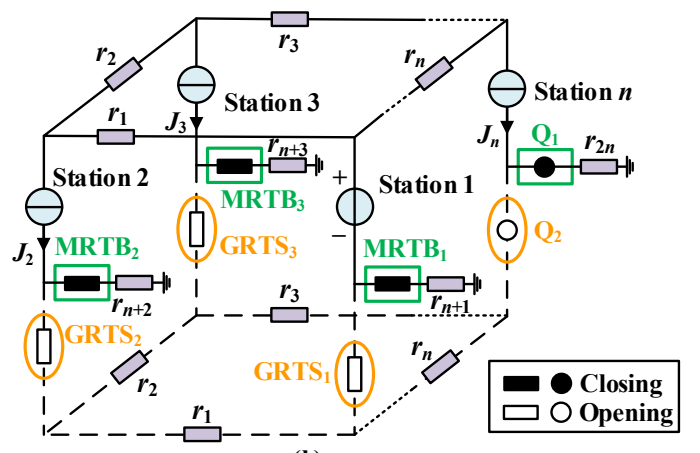

(b)

Fig. 4. Deployment Scheme 1 of MRTB and GRTS in MTDC systems. (a) Radial, (b) Ring.

Each deployment scheme of MRTB and GRTS corresponds to 2(n-1)! switching sequences of them. Based on Scheme 1, to switch from the ground return to the metallic return, $2(n-1)$ ! switching sequences of MRTB and GRTS can be generalized into two categories:

1) (i) Close $Q_{2}$. (ii) Put the ground return and metallic return circuits of all stations into parallel by closing all $(n-1)$ GRTSs. (iii) Open all $(n-1)$ MRTB in certain sequence. There are $(n-1)$ ! sequences to open $(n-1)$ MRTBs.

2) (i) Close $\mathrm{Q}_{2}$. (ii) Put the ground return and metallic return circuits of a station into parallel by closing its GRTS, and then open its MRTB. (iii) Other stations carry out the above operations in a certain sequence. There are $(n-1)$ ! sequences to carry out the above operations for $(n-1)$ stations.

Similarly, to switch from the metallic return to the ground return, there will be also $2(n-1)$ ! switching sequences of MRTB and GRTS, which can be generalized into two categories:

1) (i) Put the ground return and metallic return circuits of all stations into parallel by closing all (n-1) MRTBs. (ii) Open all (n-1) GRTS in a certain sequence. (iii) 
Open Q2. There are (n-1)! sequences to open (n-1) GRTSs.

2) (i) Put the ground return and metallic return circuits of a station into parallel by closing its MRTB, and then open its GRTS. (ii) Other stations carry out the above operations in a certain sequence. (iii) Open $\mathrm{Q}_{2}$. There are $(n-1)$ ! sequences to carry out the above operations for $(n-1)$ stations.

\section{Interrupting Current Functions of MRTB and \\ GRTS}

To switch from the ground return to the metallic return, in the order of interrupting, the interrupting currents of $(n-1)$ MRTBs are described as matrixes $\boldsymbol{B}_{1}$ to $\boldsymbol{B}_{n-1}$ :

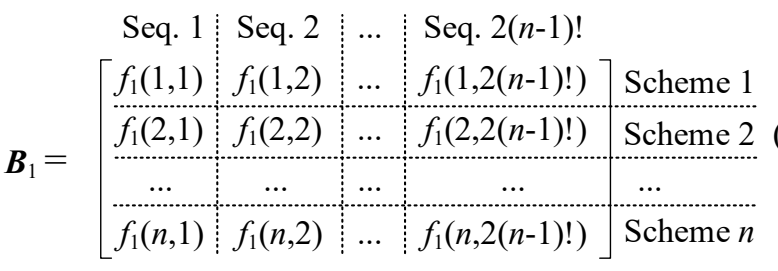

$$
\begin{aligned}
& \boldsymbol{B}_{n-1}=\left[\begin{array}{cccc}
f_{n-1}(1,1) & f_{n-1}(1,2) & \ldots & f_{n-1}(1,2(n-1) !) \\
f_{n-1}(2,1) & f_{n-1}(2,2) & \ldots & f_{n-1}(2,2(n-1) !) \\
\ldots & \ldots & \ldots & \ldots \\
f_{n-1}(n, 1) & f_{n-1}(n, 2) & \ldots & f_{n-1}(n, 2(n-1) !)
\end{array}\right]
\end{aligned}
$$

Matrixes $\boldsymbol{B}_{1}$ to $\boldsymbol{B}_{n-1}$ have $n$ rows and $2(n-1)$ ! columns. Functions $f_{s}(i, j)(1 \leq i \leq n ; 1 \leq j \leq 2(n-1) ! ; 1 \leq s \leq(n-1))$ represent the interrupting current of the $s$-th MRTB in deployment scheme $i$ and switching sequence $j$. For a radial MTDC system, functions $f_{s}(i, j)$ have $(3 n-2)$ variables: $I_{2}, I_{3}, \ldots, I_{n}, R_{1}, R_{2}, \ldots$, $R_{n-1}, R_{n}, R_{n+1}, \ldots, R_{2 n-1}$. For a ring MTDC system, functions $f_{s}(i, j)$ have $(3 n-1)$ variables: $J_{2}, J_{3}, \ldots, J_{n}, r_{1}, r_{2}, \ldots, r_{n}, r_{n+1}$, $r_{n+2}, \ldots, r_{2 n}$. Every variable of functions $f_{s}(i, j)$ varies within a certain range. The DC current of every station has a clear limit, and the resistance of the DC line or earth electrode depends on its parameters and meteorological data.

To switch from the metallic return to the ground return, in the order of interrupting, the interrupting currents of $(n-1)$ GRTSs can be described as matrixes $\boldsymbol{D}_{1}$ to $\boldsymbol{D}_{n-1}$ :

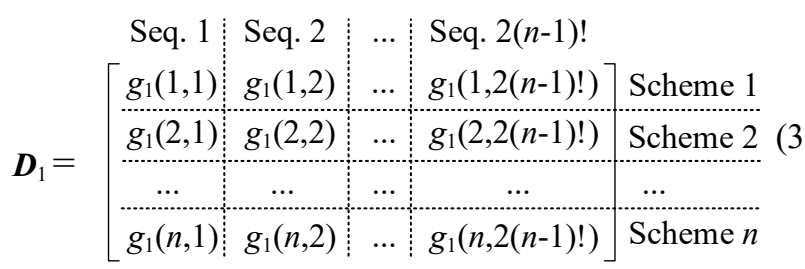

$$
\begin{aligned}
& \boldsymbol{D}_{n-1}=\left[\begin{array}{cccc}
g_{n-1}(1,1) & g_{n-1}(1,2) & \ldots & g_{n-1}(1,2(n-1) !) \\
g_{n-1}(2,1) & g_{n-1}(2,2) & \ldots & g_{n-1}(2,2(n-1) !) \\
\ldots & \ldots & \ldots & \ldots \\
g_{n-1}(n, 1) & g_{n-1}(n, 2) & \ldots & g_{n-1}(n, 2(n-1) !)
\end{array}\right]
\end{aligned}
$$

Similarly, matrixes $\boldsymbol{D}_{1}$ to $\boldsymbol{D}_{n-1}$ have $n$ rows and $2(n-1)$ ! columns. Functions $g_{s}(i, j)(1 \leq i \leq n ; 1 \leq j \leq 2(n-1) ! ; 1 \leq s \leq(n-1))$ represent the interrupting current of the $s$-th GRTS in deployment scheme $i$ and switching sequence $j$. Functions $g_{s}(i$, $j$ ) have the same number of variables as functions $f_{s}(i, j)$.

In order to achieve minimum interrupting currents of MRTB and GRTS, it is necessary to address maximum interrupting currents of MRTB and GRTS of every deployment scheme and switching sequence. $f_{s}(i, j)$ and $g_{s}(i$, $j$ ) are multivariate functions. Hence, the key issue for selecting the optimal strategy for switchover between the two operation modes is the need for a method to address maximums of interrupting current functions of MRTB and GRTS.

\section{Proposed Method to Address MaXimums of INTERRUPTING CURRENTS FUNCTIONS OF MRTB AND GRTS}

This section presents a random number calculation method to address maximums of interrupting currents functions of MRTB and GRTS, and proposes principles of selecting an optimal strategy for switchover between the ground return and metallic return operation modes.

\section{A. $\quad$ Method to Address Maximums of Interrupting Currents Functions}

Applying the nodal method of analysis and superposition theorem, expressions of functions $f_{s}(i, j)$ and $g_{s}(i, j)$ can be derived. Stems from the fact that the range of every variable in $f_{s}(i, j)$ and $g_{s}(i, j)$ is narrow, it is less likely that multivariate functions $f_{s}(i, j)$ and $g_{s}(i, j)$ contain stagnation points in their domains. In other words, it is quite possible that the partial derivatives of functions $f_{s}(i, j)$ and $g_{s}(i, j)$ are always positive or negative in their domains. Based on this feature, a random number calculation method to address maximums of functions $f_{s}(i, j)$ and $g_{s}(i, j)$ is proposed. Taking functions $f_{s}(i$, $j)$ as an example, the proposed method is illustrated in Fig. 5.

For the convenience of coding, variables of functions $f_{s}(i$, $j)$ are described as $x_{k}(k=1,2, \ldots, M$; for a radial MTDC system, $M=3 n-2$; for a ring MTDC system, $M=3 n-1$ ). The maximum value $x_{k \max }$ and the minimum value $x_{k \min }$ are consistent with the range of $x_{k}$. The maximums of functions $f_{s}(i, j)$ are marked as $F_{s}(i, j)(1 \leq i \leq n ; 1 \leq j \leq 2(n-1) ! ; 1 \leq s \leq(n-$ $1)$ ).

In order to determine the approximate range of the partial derivative $-\partial f_{s}(i, j) / \partial x_{k}$, it is recommended to generate several random numbers in the ranges of variables of $\partial f_{s}(i$, $j) / \partial x_{k}$ respectively, and then add the random numbers into it. The range of the calculation results of $\partial f_{s}(i, j) / \partial x_{k}$ is marked as $\left[\lambda_{1}, \lambda_{2}\right]$. If all random number calculation results of $\partial f_{s}(i$, $j) / \partial x_{k}$ are not less than zero $\left(\lambda_{1} \lambda_{2}>0\right.$ and $\left.\lambda_{2}>0\right)$, it is quite possible that the partial derivative is positive. That is, $f_{s}(i, j)$ increases monotonically over $x_{k}$, and $x_{k \max }$ will be the value that maximizes $f_{s}(i, j)$. Otherwise, if $f_{s}(i, j)$ decreases monotonically over $x_{k}\left(\lambda_{1} \lambda_{2}>0\right.$ and $\left.\lambda_{2}<0\right), x_{k m i n}$ will be the value that maximizes $f_{s}(i, j)$. After taking partial derivative of $f_{s}(i, j)$ over $x_{1}$, the number of variables in $\partial f_{s}(i, j) / \partial x_{1}$ is $(M-1)$, 
and they are $x_{2}, x_{3}, \ldots, x_{M}$. Given that every variable takes $N$ random numbers in its range, $\partial f_{s}(i, j) / \partial x_{1}$ needs to perform calculations for $N^{M-1}$ times. By taking partial derivatives of $f_{s}(i, j)$ and performing random number calculations cyclically, the amount of variables in $f_{s}(i, j)$ gradually decreases, and the amount of random number calculations required will be reduced. Hence, to address the maximum of any interrupting current function, the total amount of random number calculations required is at least $\sum_{m=0}^{M-1} N^{m}$.

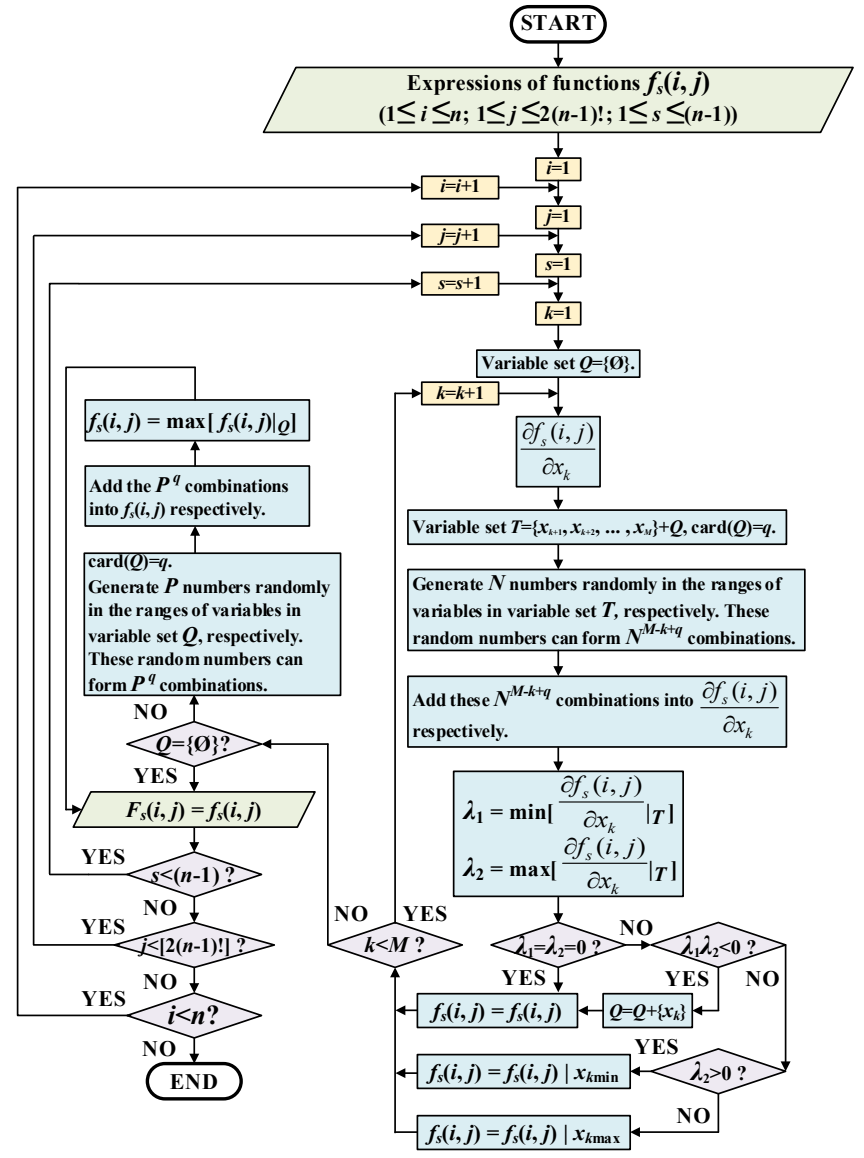

Fig. 5. A Method for addressing maximums of interrupting current functions.

If $\left[\lambda_{1}, \lambda_{2}\right]$ crosses zero $\left(\lambda_{1} \lambda_{2}<0\right)$, it is obvious that $f_{s}(i, j)$ has at least an extreme value over $x_{k}$. As a result, after completing the random number calculations for all partial derivatives, $f_{s}(i, j)$ still contains variables $(\mathrm{Q} \neq\{\varnothing\})$. For such a condition, $f_{s}(i, j)$ needs to be processed by random number calculations again. As illustrated in Fig. 5, $q$ remaining variables take $P$ random numbers in their ranges respectively, and add them into $f_{s}(i, j)$. Therefore, $f_{s}(i, j)$ needs to perform calculations for $P^{q}$ times. The maximum value of all random number calculation results is regarded as the maximum of $f_{s}(i$, $j$ ). However, according to the feature of the interrupting current functions, the probability of $\left[\lambda_{1}, \lambda_{2}\right]$ crossing zero is very small. The Maximum addressed by this method is an approximate maximum value. However, the more random numbers are taken in the range of a variable, the higher the precision of an approximate maximum value will be.
Maximums of functions $g_{s}(i, j)$ can be addressed in the same way, and mark the maximums as $G_{s}(i, j)(1 \leq i \leq n ; 1 \leq j \leq 2(n-$ $1)$ !; $1 \leq s \leq(n-1))$.

\section{B. Principles of Selecting an Optimal Strategy}

Maximum interrupting currents of MRTB under different deployment schemes and switching sequences can be represented as matrixes $\boldsymbol{B}_{1}{ }^{\prime}$ to $\boldsymbol{B}_{n-1}{ }^{\prime}$ :

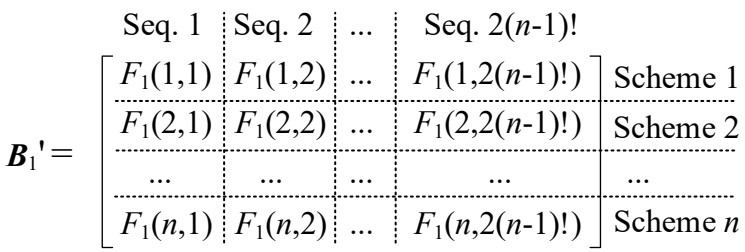

$$
\begin{aligned}
& \boldsymbol{B}_{n-1}{ }^{\prime}=\left[\begin{array}{cccc}
F_{n-1}(1,1) & F_{n-1}(1,2) & \ldots & F_{n-1}(1,2(n-1) !) \\
F_{n-1}(2,1) & F_{n-1}(2,2) & \ldots & F_{n-1}(2,2(n-1) !) \\
\ldots & \ldots & \ldots & \ldots \\
F_{n-1}(n, 1) & F_{n-1}(n, 2) & \ldots & F_{n-1}(n, 2(n-1) !)
\end{array}\right]
\end{aligned}
$$

Based on the principle in (7), the optimal deployment scheme of MRTB and GRTS is marked as Scheme $u$, and the optimal switching sequence from the ground return to the metallic return is marked as Sequence $v$.

$$
\begin{aligned}
& \min \left[F_{1}(i, j)+F_{2}(i, j)+\ldots+F_{n-1}(i, j)\right] \\
= & F_{1}(u, v)+F_{2}(u, v)+\ldots+F_{n-1}(u, v)
\end{aligned}
$$

Maximum interrupting currents of GRTS under different switching sequences can be represented as matrixes $\boldsymbol{D}_{1}{ }^{\prime}$ to $\boldsymbol{D}_{n-1}{ }^{\prime}$ :

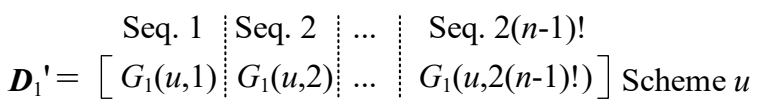

$$
\begin{aligned}
& \boldsymbol{D}_{n-1}{ }^{\prime}=\left[G_{n-1}(u, 1) \quad G_{n-1}(u, 2) \quad \ldots \quad G_{n-1}(u, 2(n-1) !)\right]
\end{aligned}
$$

Likewise, based on the principle in (10), the optimal switching sequence from the ground return to the metallic return is marked as Sequence $w$.

$$
\begin{aligned}
& \min \left[G_{1}(u, j)+G_{2}(u, j)+\ldots+G_{n-1}(u, j)\right] \\
= & G_{1}(u, w)+G_{2}(u, w)+\ldots+G_{n-1}(u, w)
\end{aligned}
$$

\section{Numerical Examples of Selecting Optimal Strategies for Switchover Between the Two Modes}

To prove the effectiveness of the proposed method of selecting an optimal strategy for switchover between the two operation modes, this section presents numerical examples. The numerical examples are based on a hybrid three-terminal radial HVDC system.

A three-terminal HVDC system and its model for analyzing the switchover between the two modes is illustrated as in Figs. 6(a) and (b). As given in Table II, for a three-terminal HVDC system, there are three deployment schemes of MRTB and GRTS. Based on Fig. 6(b), MRTB and GRTS are set up according to deployment Scheme 1, which is illustrated in Fig. 6(c). In Fig. 6(c), name the switchgears in the left station as MRTB ${ }_{I}$ and GRTS ${ }_{I}$, MRTB $_{I I}$ 


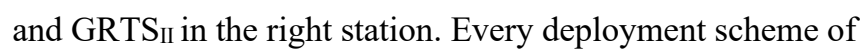
MRTB and GRTS corresponds to four switching sequences of them. Switching sequences between the ground return and the metallic return are summarized in Table III.

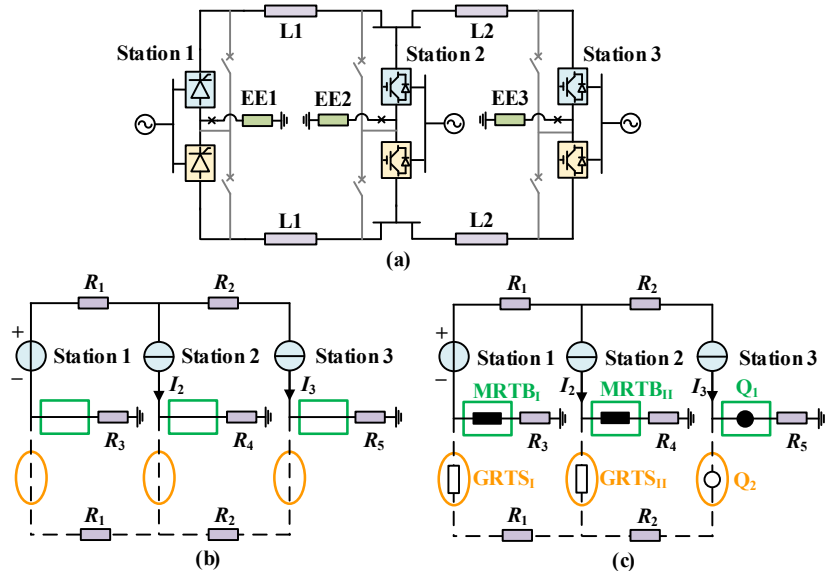

Fig. 6. A three-terminal HVDC system. (a) Topology, (b) Model for analyzing the switchover between the two modes, (c) Under deployment Scheme 1 of MRTB and GRTS.

TABLE II

DEPLOYMENT SCHEMES OF MRTB AND GRTS FOR A THREE-TERMINAL HVDC SYSTEM

\begin{tabular}{cc}
\hline \hline Deployment Schemes & Stations with MRTB and GRTS \\
\hline Scheme 1 & Station 1, Station 2 \\
Scheme 2 & Station 1, Station 3 \\
Scheme 3 & Station 2, Station 3 \\
\hline \hline
\end{tabular}

SWITCHING SEQUENCES OF MRTB AND GRTS BETWEEN THE TWO OPERATION MODES FOR A THREE-TERMINAL HVDC SYSTEM From the Ground Return to the Metallic Return

Seq. 1: close $\mathrm{Q}_{2}$, close GRTS , close GRTS $\mathrm{II}_{\text {II }}$ open MRTB $\mathrm{M}_{\mathrm{I}}$, open $\mathrm{MRTB}_{\mathrm{II}}$

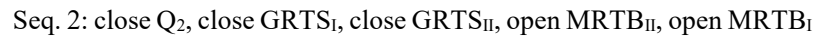
Seq. 3: close $\mathrm{Q}_{2}$, close GRTS $\mathrm{S}_{\text {I }}$, open $M R T B_{\mathrm{I}}$, close GRTS $\mathrm{S}_{\text {II }}$, open $\mathrm{MRTB}_{\mathrm{II}}$ Seq. 4: close $\mathrm{Q}_{2}$, close GRTS $\mathrm{II}_{\text {, }}$ open MRTB $\mathrm{BI}_{\text {II }}$ close GRTS $\mathrm{G}_{\mathrm{I}}$, open MRTB $\mathrm{B}_{\mathrm{I}}$ From the Metallic Return to the Ground Return

Seq. 1:close MRTB ${ }_{\mathrm{I}}$, close $\mathrm{MRTB}_{\mathrm{II}}$, open GRTS , open GRTS $\mathrm{II}_{\mathrm{I}}$, open $\mathrm{Q}_{2}$ Seq. 2:close MRTB $\mathrm{B}_{\mathrm{I}}$, close MRTB $\mathrm{B}_{\mathrm{II}}$, open GRTS II, open GRTS $\mathrm{S}_{\mathrm{I}}$, open $\mathrm{Q}_{2}$ Seq. 3:close MRTB $\mathrm{B}_{\mathrm{I}}$, open GRTS , close MRTB ${ }_{\text {II }}$, open GRTS $\mathrm{II}_{\text {II }}$, open $\mathrm{Q}_{2}$ Seq. 4:close MRTB ${ }_{\text {II }}$, open GRTS II, close MRTB , open GRTS , open $\mathrm{Q}_{2}$

From the ground return to the metallic return, in the order of interrupting, the interrupting currents of two MRTBs can be described as matrixes $\boldsymbol{B}_{1}$ and $\boldsymbol{B}_{2}$ :

$$
\begin{gathered}
\boldsymbol{B}_{1}=\left[\begin{array}{c:c:c:c}
\text { Seq. 1 } & \text { Seq. 2 } & \text { Seq. 3 } & \text { Seq. 4 } \\
f_{1}(1,1) & f_{1}(1,2) & f_{1}(1,3) & f_{1}(1,4) \\
\hdashline f_{1}(2,1) & f_{1}(2,2) & f_{1}(2,3) & f_{1}(2,4) \\
\hdashline f_{1}(3,1) & f_{1}(3,2) & f_{1}(3,3) & f_{1}(3,4)
\end{array}\right] \text { Scheme } 1 \\
\text { Scheme 2 } \\
\boldsymbol{B}_{2}=\left[\begin{array}{llll}
f_{2}(1,1) & f_{2}(1,2) & f_{2}(1,3) & f_{2}(1,4) \\
f_{2}(2,1) & f_{2}(2,2) & f_{2}(2,3) & f_{2}(2,4) \\
f_{2}(3,1) & f_{2}(3,2) & f_{2}(3,3) & f_{2}(3,4)
\end{array}\right]
\end{gathered}
$$

Interrupting current functions $f_{s}(i, j) \quad(i=1,2,3 ; j=1,2,3,4$; $s=1,2)$ have seven variables. The expressions of functions $f_{s}(i$, $j)$ are given as (A1) (A12) in Appendix A. $F_{s}(i, j)(i=1,2,3$; $j=1,2,3,4 ; s=1,2)$ are maximums of functions $f_{s}(i, j) . F_{s}(i, j)$ can be addressed by the random number calculation method. The method can be implemented using MATLAB. Applying the data in Table $\mathrm{IV}, F_{s}(i, j)$ are obtained and presented as below:

$$
\begin{aligned}
& \boldsymbol{B}_{1}{ }^{\prime}=\left[\begin{array}{llll}
3.8161 & 1.4699 & 4.1705 & 2.4048 \\
3.8161 & 2.6488 & 4.0029 & 3.0975 \\
1.4699 & 2.6488 & 1.0885 & 2.3768
\end{array}\right] \\
& \boldsymbol{B}_{2}{ }^{\prime}=\left[\begin{array}{llll}
1.5222 & 3.1014 & 1.5222 & 3.1014 \\
1.5222 & 2.9651 & 1.5222 & 2.9651 \\
3.1014 & 2.9651 & 3.1014 & 2.9651
\end{array}\right] \\
& \min \left[F_{1}(i, j)+F_{2}(i, j)\right]=F_{1}(3,3)+F_{2}(3,3) \\
&=1.0885+3.1014 \\
&=4.1899 \\
& \text { TABLE IV }
\end{aligned}
$$

RANGES OF PARAMETERS OF A THREE-TERMINAL HVDC SYSTEM

\begin{tabular}{ccc}
\hline \hline Parameters & Maximums & Minimums \\
\hline$I_{2}$ & $1.875 \mathrm{kA}$ & $0.1875 \mathrm{kA}$ \\
$I_{3}$ & $3.125 \mathrm{kA}$ & $0.3125 \mathrm{kA}$ \\
$R_{1}$ & $4.3694 \Omega$ & $3.4369 \Omega$ \\
$R_{2}$ & $4.3719 \Omega$ & $3.4436 \Omega$ \\
$R_{3}$ & $0.7434 \Omega$ & $0.5518 \Omega$ \\
$R_{4}$ & $3.4477 \Omega$ & $2.4469 \Omega$ \\
$R_{5}$ & $2.9703 \Omega$ & $2.1563 \Omega$ \\
\hline \hline
\end{tabular}

As presented in (15), the optimal deployment scheme of MRTB and GRTS is Scheme 3, and the optimal switching sequence of MRTB and GRTS from the ground return to the metallic return is Sequence 3.

From the metallic return to the ground return, in the order of interrupting, the interrupting currents of two GRTSs can be described as matrixes $\boldsymbol{D}_{1}$ and $\boldsymbol{D}_{2}$ :

$$
\begin{aligned}
& \begin{array}{l:l:l:l}
\text { Seq. } 1 & \text { Seq. } 2 & \text { Seq. } 3 & \text { Seq. } 4
\end{array} \\
& \boldsymbol{D}_{1}=\left[\begin{array}{l:l:l:l}
g_{1}(3,1) & g_{1}(3,2) & g_{1}(3,3) & \left.g_{1}(3,4)\right] \text { Scheme } 3
\end{array}\right. \\
& \boldsymbol{D}_{2}=\left[\begin{array}{llll}
g_{2}(3,1) & g_{2}(3,2) & g_{2}(3,3) & g_{2}(3,4)
\end{array}\right]
\end{aligned}
$$

The expressions of functions $g_{s}(3, j)(j=1,2,3,4 ; s=1,2)$ are given as (A13) (A16) in Appendix A. $G_{s}(3, j) \quad(j=1,2,3,4$; $s=1,2)$ are maximums of functions $g_{s}(3, j)$, which are shown as follows:

$$
\begin{aligned}
& \boldsymbol{D}_{1}{ }^{\prime}=\left[G_{1}(3,1) \quad G_{1}(3,2) \quad G_{1}(3,3) \quad G_{1}(3,4)\right] \\
& =\left[\begin{array}{llll}
1.2168 & 1.2444 & 1.7769 & 1.0346
\end{array}\right] \\
& \boldsymbol{D}_{2}{ }^{\prime}=\left[G_{2}(3,1) \quad G_{2}(3,2) \quad G_{2}(3,3) \quad G_{2}(3,4)\right] \\
& =\left[\begin{array}{llll}
1.2270 & 1.3347 & 1.2270 & 1.3347
\end{array}\right] \\
& \min \left[G_{1}(3, j)+G_{2}(3, j)\right]=G_{1}(3,4)+G_{2}(3,4) \\
& =1.0346+1.3347 \\
& =2.3694
\end{aligned}
$$

As presented in (20), the optimal switching sequence of MRTB and GRTS from the metallic return to the ground return is Sequence 4.

\section{Simulations of the Optimal Switchover Strategies}

This section presents simulations of the optimal strategies 
for switchover between the ground return and metallic return modes in the numerical examples. The three-terminal HVDC network is illustrated in Fig. 7. Define the parameters $\left(I_{2}, I_{3}\right.$, $\left.I_{4}, R_{1}, R_{2}, R_{3}, R_{4}, R_{5}\right)$ in Fig. 7 as $(1.875 \mathrm{kA}, 3.125 \mathrm{kA}, 6.4 \mathrm{kA}$, $3.4369 \Omega, 3.4436 \Omega, 0.5518 \Omega, 3.4477 \Omega, 2.9703 \Omega$ ). DC currents of the switchgears in three stations during the switchover between two modes are illustrated in Fig. 8.

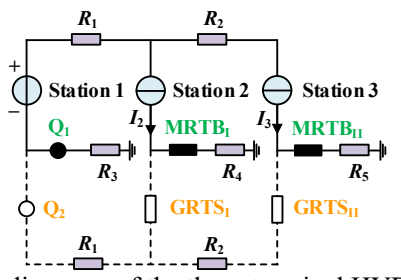

Fig. 7. Connection diagrams of the three-terminal HVDC network.

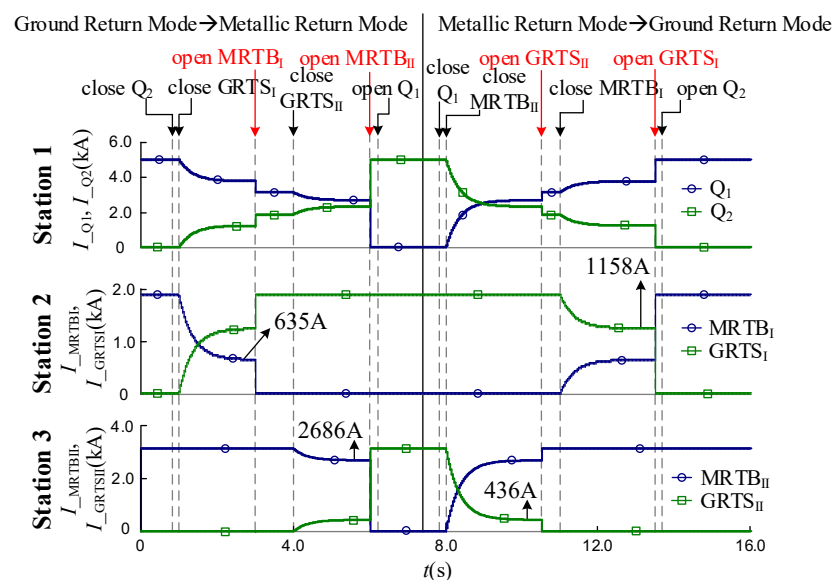

Fig. 8. DC currents of the switchgears in three stations during the switchover between the ground return and metallic return modes (Case 1).

According to section IV, the interrupting currents of MRTB and GRTB are all within the interrupting capacities.

\section{CONCLUSION}

This paper proposes a method to select the optimal strategy for the switchover between the ground return and metallic return operation modes of MTDC systems. For an $n$-terminal HVDC system (radial or ring), there are $n$ deployment schemes of MRTB and GRTS, and 2(n-1)! switching sequences from the ground return to metallic return modes, and vice versa. Thanks to the proposed optimal strategy, interrupting capacities of MRTB and GRTS can be the smallest and therefore, the capital costs can be minimized.

A method has been proposed to address maximums of the interrupting current functions of MRTB and GRTS under every scheme and sequence. The method verifies the monotonic characteristics of the interrupting current function over one of its variables by performing the random number calculations of its partial derivative. Therefore, a value of the variable that maximizes the function is determined. By performing random number calculations for all variables, a maximum of the function is obtained. Thus, by comparison, an optimal strategy for the switchover with minimum interrupting currents of switchgears can be selected.

The optimization method proposed in this paper provides a strong reference for the future multi-terminal HVDC transmission system to select the strategy for switchover between the ground return and the metallic return operation modes.

\section{APPENDIX A}

$$
\begin{aligned}
& \left\{\begin{array}{c}
f_{1}(1,1)=\frac{\left(R_{2}+R_{4}+R_{5}\right) R_{1} I_{2}+\left[\left(R_{2}+R_{4}+R_{5}\right) R_{1}+R_{2} R_{4}\right] I_{3}}{\left(R_{1}+R_{3}+R_{4}\right)\left(R_{2}+R_{4}+R_{5}\right)-R_{4}{ }^{2}} \\
f_{2}(1,1)=-\frac{R_{2} I_{3}}{R_{2}+R_{4}+R_{5}}
\end{array}\right. \\
& \left\{f_{1}(1,2)=\frac{\left(R_{2}+R_{5}\right) R_{1} I_{2}+\left(R_{1} R_{5}-R_{2} R_{3}\right) I_{3}}{\left(R_{1}+R_{3}+R_{4}\right)\left(R_{2}+R_{4}+R_{5}\right)-R_{4}{ }^{2}}\right. \\
& f_{2}(1,2)=\frac{R_{1} I_{2}+\left(R_{1}+R_{2}\right) I_{3}}{R_{1}+R_{2}+R_{3}+R_{5}} \\
& \left\{f_{1}(1,3)=\frac{\left(R_{1}+R_{2}+R_{5}\right) I_{2}+\left(R_{1}+R_{2}\right) I_{3}}{R_{1}+R_{2}+R_{3}+R_{5}}\right. \\
& f_{2}(1,3)=-\frac{R_{2} I_{3}}{R_{2}+R_{4}+R_{5}} \\
& \left\{\begin{array}{l}
f_{1}(1,4)=\frac{\left(R_{2}+R_{5}\right) I_{2}+R_{5} I_{3}}{R_{2}+R_{4}+R_{5}} \\
f_{2}(1,4)=\frac{R_{1} I_{2}+\left(R_{1}+R_{2}\right) I_{3}}{R_{1}+R_{2}+R_{3}+R_{5}}
\end{array}\right. \\
& \left\{f_{1}(2,1)=\frac{\left(R_{2}+R_{4}+R_{5}\right) R_{1} I_{2}+\left[\left(R_{2}+R_{4}+R_{5}\right) R_{1}+R_{2} R_{4}\right] I_{3}}{\left(R_{1}+R_{3}+R_{4}\right)\left(R_{2}+R_{4}+R_{5}\right)-R_{4}{ }^{2}}\right. \\
& f_{2}(2,1)=\frac{R_{2} I_{3}}{R_{2}+R_{4}+R_{5}} \\
& \left\{f_{1}(2,2)=\frac{R_{1} R_{4} I_{2}+\left[\left(R_{1}+R_{3}+R_{4}\right) R_{2}+R_{1} R_{4}\right] I_{3}}{\left(R_{1}+R_{3}+R_{4}\right)\left(R_{2}+R_{4}+R_{5}\right)-R_{4}^{2}}\right. \\
& f_{2}(2,2)=\frac{R_{1} I_{2}+R_{1} I_{3}}{R_{1}+R_{3}+R_{4}} \\
& \left\{f_{1}(2,3)=\frac{R_{1} I_{2}+\left(R_{1}+R_{4}\right) I_{3}}{R_{1}+R_{3}+R_{4}}\right. \\
& f_{1}(2,3)=\frac{R_{2} I_{3}}{R_{2}+R_{4}+R_{5}} \\
& \left\{f_{1}(2,4)=\frac{R_{4} I_{2}+\left(R_{2}+R_{4}\right) I_{3}}{R_{2}+R_{4}+R_{5}}\right. \\
& f_{2}(2,4)=\frac{R_{1} I_{2}+R_{1} I_{3}}{R_{1}+R_{3}+R_{4}} \\
& \left\{f_{1}(3,1)=\frac{\left(R_{2}+R_{5}\right) R_{1} I_{2}+\left(R_{1} R_{5}-R_{2} R_{3}\right) I_{3}}{\left(R_{1}+R_{3}+R_{4}\right)\left(R_{2}+R_{4}+R_{5}\right)-R_{4}{ }^{2}}\right. \\
& f_{2}(3,1)=\frac{R_{1} I_{2}+\left(R_{1}+R_{2}\right) I_{3}}{R_{1}+R_{2}+R_{3}+R_{5}} \\
& \left\{f_{1}(3,2)=\frac{R_{1} R_{4} I_{2}+\left[\left(R_{1}+R_{3}+R_{4}\right) R_{2}+R_{1} R_{4}\right] I_{3}}{\left(R_{1}+R_{3}+R_{4}\right)\left(R_{2}+R_{4}+R_{5}\right)-R_{4}^{2}}\right. \\
& f_{2}(3,2)=\frac{R_{1} I_{2}+R_{1} I_{3}}{R_{1}+R_{3}+R_{4}} \\
& f_{1}(3,3)=\frac{R_{1} I_{2}-R_{3} I_{3}}{R_{1}+R_{3}+R_{4}} \\
& f_{2}(3,3)=\frac{R_{1} I_{2}+\left(R_{1}+R_{2}\right) I_{3}}{R_{1}+R_{2}+R_{3}+R_{5}}
\end{aligned}
$$




$$
\begin{gathered}
\left\{\begin{array}{c}
f_{1}(3,4)=\frac{-R_{3} I_{2}+\left(R_{1}+R_{2}\right) I_{3}}{R_{1}+R_{2}+R_{3}+R_{5}} \\
f_{2}(3,4)=\frac{R_{1} I_{2}+R_{1} I_{3}}{R_{1}+R_{3}+R_{4}}
\end{array}\right. \\
\left\{\begin{array}{c}
g_{1}(3,1)=\frac{\left[\left(R_{2}+R_{4}+R_{5}\right)\left(R_{3}+R_{4}\right)+\left(R_{1}-R_{4}\right) R_{4}\right] I_{2}-\left(R_{1} R_{5}-R_{2} R_{3}\right) I_{3}}{\left(R_{1}+R_{3}+R_{4}\right)\left(R_{2}+R_{4}+R_{5}\right)-R_{4}^{2}} \\
g_{2}(3,1)=\frac{R_{3} I_{2}+\left(R_{3}+R_{5}\right) I_{3}}{R_{1}+R_{2}+R_{3}+R_{5}} \\
g_{1}(3,2)=\frac{-R_{1} R_{4} I_{2}+\left[\left(R_{1}+R_{3}+R_{4}\right) R_{5}+R_{3} R_{4}\right] I_{3}}{\left(R_{1}+R_{3}+R_{4}\right)\left(R_{2}+R_{4}+R_{5}\right)-R_{4}^{2}} \\
g_{2}(3,2)=\frac{\left(R_{3}+R_{4}\right) I_{2}+R_{3} I_{3}}{R_{1}+R_{3}+R_{4}} \\
g_{1}(3,3)=\frac{\left(R_{3}+R_{4}\right) I_{2}-R_{1} I_{3}}{R_{1}+R_{3}+R_{4}} \\
g_{2}(3,3)=\frac{R_{3} I_{2}+\left(R_{3}+R_{5}\right) I_{3}}{R_{1}+R_{2}+R_{3}+R_{5}} \\
g_{1}(3,4)=\frac{-R_{1} I_{2}+\left(R_{3}+R_{5}\right) I_{3}}{R_{1}+R_{2}+R_{3}+R_{5}} \\
g_{2}(3,4)=\frac{\left(R_{3}+R_{4}\right) I_{2}+R_{3} I_{3}}{R_{1}+R_{3}+R_{4}}
\end{array}\right. \\
\end{gathered}
$$

\section{REFERENCES}

[1] E. Kontos, R. T. Pinto, S. Rodrigues and P. Bauer, "Impact of HVDC Transmission System Topology on Multiterminal DC Network Faults," in IEEE Trans. Power Del., vol. 30, no. 2, pp. 844-852, April 2015.

[2] G. Li, J. Liang, F. Ma, C. E. Ugalde-Loo and H. Liang, "Analysis of Single-Phase-to-Ground Faults at the Valve-Side of HB-MMCs in HVDC Systems," IEEE Trans. Ind. Electron., vol. 66, no. 3, pp. 24442453, March 2019.

[3] S. Beckler, J. Lehner, A. Arnold, A. K. Kamga, K. Frey and K. Rudion, "DC Fault Currents for FB-MMC HVDC with Bipolar Configuration," in International ETG Congress 2015; Die Energiewende - Blueprints for the new energy age, Bonn, Germany, 2015.

[4] A. L. Courts, J. J. Vithayathil, N. G. Hingorani, J. W. Porter, J. G. Gorman and C. W. Kimblin, "A New DC Breaker Used as Metallic Return Transfer Breaker,” in IEEE Trans. Power App. Syst., vol. PAS101, no. 10, pp. 4112-4121, Oct. 1982.

[5] J. Hao, W. Teng, Y. Zhang and W. Liu, "Research on Distribution Characteristics of DC Potential Near the UHVDC Grounding Electrode," in IEEE Access, vol. 8, pp. 122360-122365, 2020.

[6] B. Zhang et al., "DC Current Distribution in Both AC Power Grids and Pipelines Near HVDC Grounding Electrode Considering Their Interaction," in IEEE Trans. Power Del., vol. 34, no. 6, pp. 2240-2247, Dec. 2019.

[7] W. Jiang, G. N. Wu and H. L. Wang, "Calculation of DC Grounding Current Distribution by UHVDC Mono-Polar Operation with Ground Return," in 2008 IEEE/PES Transmission and Distribution Conference and Exposition, Chicago, IL, USA, 2008.
[8] M. Backman, L. Liljestrand, F. Rafatnia and R. Du, "Passive DC neutral breaker for bipolar HVDC schemes," in 2017 4th International Conference on Electric Power Equipment - Switching Technology (ICEPE-ST), Xi'an, China, 2017.

[9] Z. Liu, "Uhv converter station and uhvdc electrical equipment," in Ultra-High voltage ac/dc grids, New York, NY, USA: Academic Press, 2015, pp. 517-521.

[10] The China Electric Power Research Institute, "Uhvdc system overvoltage and insulation coordination," in Uhv transmission technology, New York, NY, USA: Academic Press, 2018, pp. 521-557.

[11] E. Kontos, G. Tsolaridis, R. Teodorescu and P. Bauer, "On DC Fault Dynamics of MMC-Based HVdc Connections," in IEEE Trans. Power Del., vol. 33, no. 1, pp. 497-507, Feb. 2018.

[12] J. M. S. Fuentes-Burruel and E. L. Moreno-Goytia, "CIGRE B4 DC Grid Test System: Performance of a DC Hybrid Breaker during a poleto-ground DC Bus Fault," in 2019 IEEE International Autumn Meeting on Power, Electronics and Computing (ROPEC), Ixtapa, Mexico, 2019.

[13] G. Li et al., "Feasibility and Reliability Analysis of LCC DC Grids and LCC/VSC Hybrid DC Grids," in IEEE Access, vol. 7, pp. 22445-22456, 2019.

[14] C. Guo, A. Zheng and C. Zhao, "Coupling Effect Investigation of Series-Connected Converters in MMC-Based Ultra-HVDC Station," in IEEE Journal of Emerging and Selected Topics in Power Electronics, vol. 8, no. 4, pp. 3307-3317, Dec. 2020.

[15] Y. Li, R. Cao, W. Huang, G. Li, Z. Guo and T. Liu, "Design and Simulation for the Control and Protection of Hybrid LCC-VSC MTDC System," in 2019 IEEE 3rd International Electrical and Energy Conference (CIEEC), Beijing, China, 2019.

[16] Y. Song, L. Zhu, Z. Zhao and T. Zhang, "Simulation of the transient characteristics in HVDC scheme during the operation of MRTB," in 2016 IEEE International Power Modulator and High Voltage Conference (IPMHVC), San Francisco, CA, USA, 2016.

[17] J. Li, R. Hu, Y. Wen and Y. Li, "Analysis of an abnormal GRTS transfer in DC double circuit transmission system," in 2016 IEEE PES AsiaPacific Power and Energy Engineering Conference (APPEEC), Xi'an, China, 2016.

[18] Y. Li, J. Lian, J. Jin, Z. Liu, Z. Zhang and J. Cao, "Testing Research on Auxiliary Circuit Oscillation Characteristic Parameters for High Voltage DC Transfer Switch," in 2018 IEEE 3rd Advanced Information Technology, Electronic and Automation Control Conference (IAEAC), Chongqing, China, 2018.

[19] H. Ying, X. Qingming, Z. Xiaobin, L. yuxin and G. Long, "Research on Fast Conversion Method of Metallic Return to Earth Return Operation mode in HVDC System," in 2018 International Conference on Power System Technology (POWERCON), Guangzhou, China, 2018.

[20] B. Zhang, W. Deng, T. Wang, J. Chen, Y. Teng and X. Wu, "Electromagnetic transient modeling and simulation of large-scale HVDC power grid with all primary devices," in 2016 IEEE International Conference on Power and Renewable Energy (ICPRE), Shanghai, China, 2016.

[21] Y. Chen et al., "Analysis of Transfer Operation between Metallic and Ground Return Configurations for Multi-terminal HVDC Systems," in 2019 4th IEEE Workshop on the Electronic Grid (eGRID), Xiamen, China, 2019. 